\title{
Mechanical characterization of a dielectric elastomer microactuator with ion-implanted electrodes
}

\author{
S. Rosset, M. Niklaus, P. Dubois, H.R. Shea
}

The following document is a post-print of the article published in Sensors and Actuators A 144 (2008)

185-193. The original article can be accessed at http://dx.doi.org/10.1016/j.sna.2007.12.030 


\title{
Mechanical Characterization of a Dielectric Elastomer Microactuator With Ion-Implanted Electrodes
}

\author{
S. Rosset*, M. Niklaus, P. Dubois, H. R. Shea \\ Microsystems for Space Technologies Laboratory, Ecole Polytechnique Fédérale de \\ Lausanne, Switzerland
}

\begin{abstract}
We report on the mechanical characterization and modeling of a non-prestretched dielectric elastomer diaphragm microactuator with ion-implanted electrodes under the influence of a distributed load (pressure). Thin PDMS membranes (30 $\mu \mathrm{m}$ thick, 2-3 mm diameter) were implanted on both side with gold ions by Filtered Cathodic Vacuum Arc and bonded on silicon chips with through-holes. A voltage applied between the implanted electrodes creates a compressive stress in the dielectric and causes the membrane to buckle and form a bump whose height depends on the mechanical properties of the electroactive compound, the voltage and the force applied on the membrane. Maximum unloaded displacements up to $7 \%$ of the membrane's lateral dimensions were achieved, which was reduced to $3 \%$ when a distributed force of $1 \mathrm{kPa}$ was applied on the membrane. The maximum mechanical work obtained by the actuators is in the range of $0.3 \mu \mathrm{J}$. An analytical model was developped to calculate the displacement of the DEAs based on their mechanical and geometrical properties, voltage and applied force. The model shows very good agreement with the measurements and allows accurate performance prediction and dimensioning of such actuators.
\end{abstract}

Key words: Dielectric Electroactive Polymer Actuators, Ion Implantation, Electrostatic Actuation, Membrane, Artificial Muscles, DEAP

* Corresponding author: rue Jaquet-Droz 1, cp 526, 2002 Neuchâtel, Switzerland Email address: samuel.rosset@a3.epfl.ch (S. Rosset). 


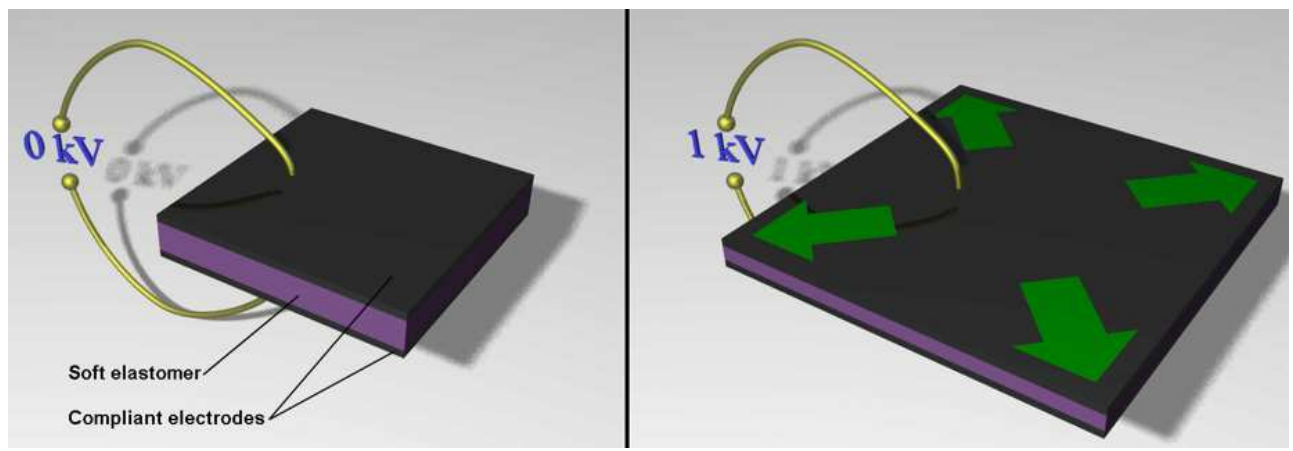

Fig. 1. Dielectric EAP (DEAP) principle. When a voltage is applied to the electrodes (typically up to $1 \mathrm{kV}$ ), the electrostatic pressure squeezes the elastomer dielectric (right side). The volume of the dielectric being quasi constant, the whole structure stretches in the case of free boundary conditions (from [6]).

\section{Introduction}

Electroactive Polymers (EAPs) actuators have attracted wide interest for the last 15 years due to their large strain capabilities (up to several hundred percents) coupled with reasonable output actuation pressure (about $1 \mathrm{kPa}$ ) and low density $\left(\rho \approx 1000 \mathrm{~kg} / \mathrm{m}^{3}\right)$, which gives them properties similar to those of natural muscles [1]. Among the two main classes of EAPs (Electronic and Ionic [2]), Dielectric Elastomer Actuators (DEAs) have the advantage of being electrostatically driven: they are low-power actuators, require no power to hold a position and have a relatively fast response time. DEAs consist of a soft dielectric (typically Polydimethysiloxane (PDMS) or Acrylic) sandwiched between two conductive and compliant electrodes. When a voltage is applied between the electrodes, a compressive stress is generated inside the dielectric which is squeezed. As elastomers keep their volume constant during deformation (Poisson coefficient close to 0.5 ), the thickness' decrease causes the surface to expand in the case of free boundary conditions (Fig. 1). Most of the time, electrodes are made a) with carbon powder mixed with unpolymerized elastomer or sprayed onto the cured polymer, b) with carbon grease applied with a paintbrush, or c) with conducting polymers [3-5]. This is a major drawback for the miniaturization of DEAs due to the impossibility to pattern micronscale conductive electrodes and the incompatibility of carbon particles with a clean-room environment. Attempts to use conventional thin-film deposition methods (sputtering, evaporation) leads to a dramatic decrease of performance due to the stiffening of the structure caused by the high Young's Moduli of metals compared to that of elastomer (up to five orders of magnitude) $[6,7]$.

We use low energy Filtered Cathodic Vacuum Arc (FCVA) metal ion implantation to create compliant electrodes at the surface of the PDMS. This creates a conductive layer in the first few nanometers of the PDMS' surface, without stiffening it too much. This technique can easily be used to make patterned 
electrodes by using a photoresist or steel shadow mask during the implantation. We have shown the applicability of FCVA implantation to make compliant electrodes for miniaturized DEAs [8]. A measurement of the mechanical properties of titanium-implanted PDMS layers showed a limited implantationinduced increase in stiffness (between 10\% and 100\% depending on dose) and a slight stress decrease, whereas thin sputtered gold layers $(\approx 8 \mathrm{~nm})$ lead to an increase in stiffness of about $400 \%$ coupled with an important (40\%) tensile stress increase in the membrane [6].

In this paper we present the first full mechanical characterization of a mmsize membrane DEA whose electrodes are made by gold ions implantation, in terms of displacement and mechanical work. We also present a simplified analytical model to predict the out-of-plane deflection of circular and square EAP membranes under the action of a distributed force. This model, which shows excellent agreement with the data points, can be used for dimensioning DEAs in order to obtain a given displacement and force output. In $\S 5$, the model will be applied to the calculation of a DEAP-based micropump as an illustration.

\section{Concept and Model}

In its most basic configuration, DEAP actuators consist of a dielectric layer sandwiched between two compliant electrodes. Applying a voltage between the electrodes creates an actuation pressure $\left(\sigma_{e}\right)$ which is proportional to the square of the applied electric field [3]:

$$
\sigma_{e}=-\epsilon_{0} \cdot \epsilon_{r} \cdot \frac{V^{2}}{t^{2}}(\mathrm{~Pa})
$$

where $\epsilon_{0}$ and $\epsilon_{r}$ are respectively the vacuum permittivity and relative permittivity of the elastomer, $V$ is the applied voltage, and $t$ is the thickness of the dielectric membrane. This pressure is twice the one of conventional electrostatic actuators due to the fact that the electrodes are compliant and expand during deformation. If the boundary conditions are free and the Young's Modulus of the elastomer is known, the resulting thickness compression and area expansion can be calculated from Eq. 1, taking into account the very high bulk modulus of elastomers leading to Poisson coefficient of 0.5.

Our membranes have fixed boundary conditions and cannot laterally expand when a voltage is applied. The membrane is instead in an isostatic stress state, in which the electrically-induced vertical (z axis) stress $\sigma_{e}$ is integrally transmitted on the lateral $x$ and $y$ axes. In case the fabrication process creates lateral residual stress in the membrane, or if an intentional prestretch has been 
applied, the electrostatic pressure adds to the residual stress in the $x$ and $y$ directions [9]:

$$
\begin{array}{r}
\sigma_{z}=\sigma_{e}=-\epsilon_{0} \cdot \epsilon_{r} \cdot \frac{V^{2}}{t^{2}} \\
\sigma_{x}=\sigma_{y}=\sigma_{0}+\sigma_{e}=\sigma_{0}-\epsilon_{0} \cdot \epsilon_{r} \cdot \frac{V^{2}}{t^{2}}
\end{array}
$$

where $\sigma_{x, y, z}$ represents the stress in the membrane's three principal axes and $\sigma_{0}$ represents the initial in-plane residual stress, which is assumed equal in both $x$ and $y$ direction. If the voltage is high enough, the lateral stress will reach the compressive buckling stability limit. For circular membranes with clamped edges, this critical stress value at which buckling occurs and out-ofplane deflection is observed is given by [10]:

$$
\sigma_{c r}=-1.22 \frac{E}{1-\nu^{2}}\left(\frac{t}{r}\right)^{2}
$$

where $E$ is the Young modulus, $\nu$ the Poisson coefficient ( 0.5 for elastomers), $t$ and $r$ the thickness and radius of the membrane. Typical values of critical stress for circular elastomeric membranes are calculated with Eq. 3 and shown in Fig. 2. The buckling voltage $V_{b}$ (i.e. the voltage needed to reach the buckling threshold) is obtained by combining Eq. 2 and 3:

$$
V_{b}=\frac{t}{\sqrt{\epsilon_{0} \cdot \epsilon_{r}}} \sqrt{\sigma_{0}+1.22 \frac{E}{1-\nu^{2}}\left(\frac{t}{r}\right)^{2}} .
$$

Depending on the value of the initial stress $\sigma_{0}$, which can be induced by the fabrication process $(10-100 \mathrm{kPa})$ or intentionally applied by prestretching the membrane (up to 2-10 MPa), most of the electrical energy will be used to cancel the tensile stress, compared to the few kilopascals needed to go from the zero stress state to the buckling limit. Consequently, if free-strain (i.e. without an external applied force) out-of-plane motion of the DEA membrane is desirable, care should be taken to minimize the tensile stress in the membrane in order to lower the buckling voltage. Acrylic-based DEAs, which need to be heavily prestreched are therefore not suitable for this application, for the dielectric breakdown limit of the elastomer would be reached before the buckling threshold. We use PDMS (Nusil CF19-2186) that we apply by spin-coating and cure at low temperature $\left(6^{\circ} \mathrm{C}\right.$ or room temperature $)$ to reduce residual stress in order to achieve low buckling threshold. In practice, the buckling threshold is not sharply defined because the membranes are not initially perfectly flat: initial deformations help promote vertical displacement for voltages below $V_{b}$. 


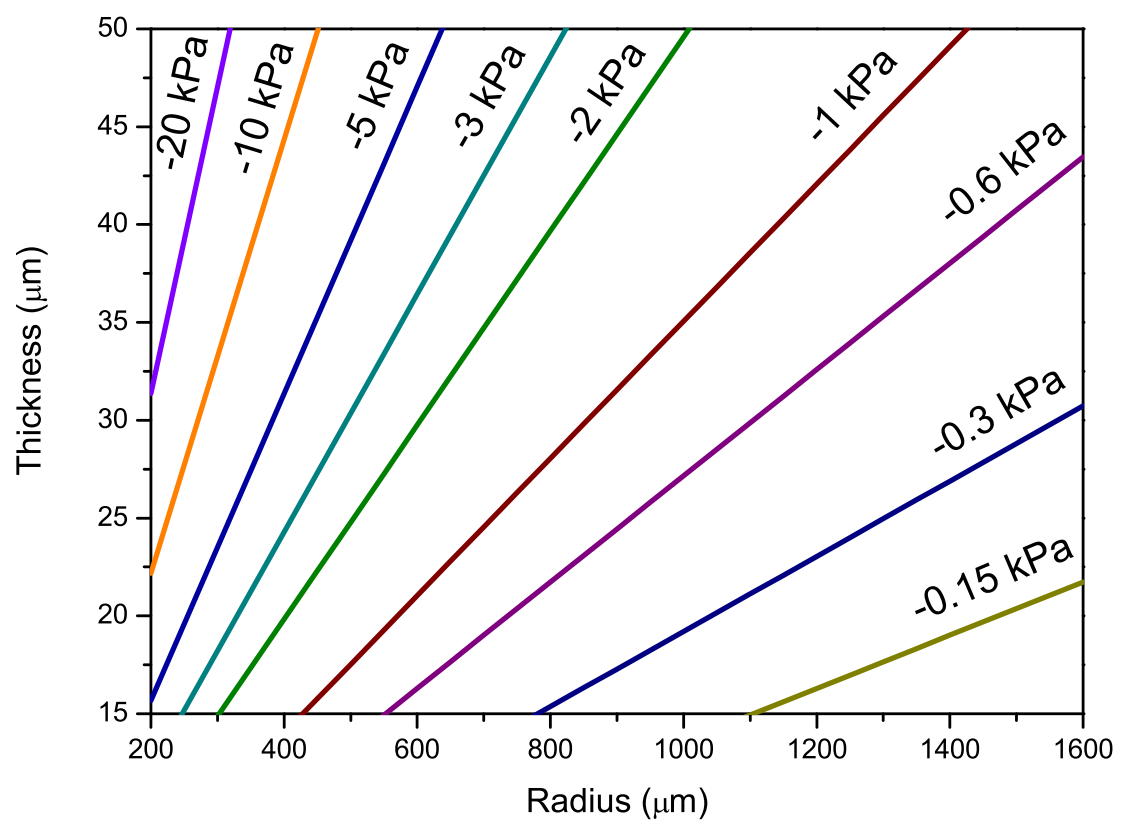

Fig. 2. Calculated critical buckling stress for circular membranes of different radius and thickness for an elastomer with $\mathrm{E}=0.5 \mathrm{MPa}$, and a Poisson coefficient of 0.5

The application of an external distributed force on the surface of the EAP membrane will create an out-of-plane deformation whose amplitude will depend on the magnitude of the distributed force, and voltage. Because the pressure-induced deformation is large due to the low Young's Modulus of elastomer, the deformation will be dominated by the stretching of the membrane, rather than bending. The deformation shape for a clamped circular membrane is assumed to be a portion of sphere, and its out-of-plane deflection is defined by [11] :

$$
p=(1-0.24 \nu)\left(\frac{8 E}{3-3 \nu}\right)\left(\frac{t}{r^{4}}\right) z^{3}+4\left(\frac{\sigma_{x, y} t}{r^{2}}\right) z,
$$

where $p$ is the applied pressure $\sigma_{x, y}$ is the voltage-dependent in-plane stress that can be substituted by Eq. 2 and $z$ is the vertical deflection of the membrane's center. The deformation into a portion of sphere creates an area expansion and thickness decrease due to the constant volume. The thickness for a given deformation $z$ is given by:

$$
t=\frac{r^{2} t_{0}}{r^{2}+z^{2}}
$$




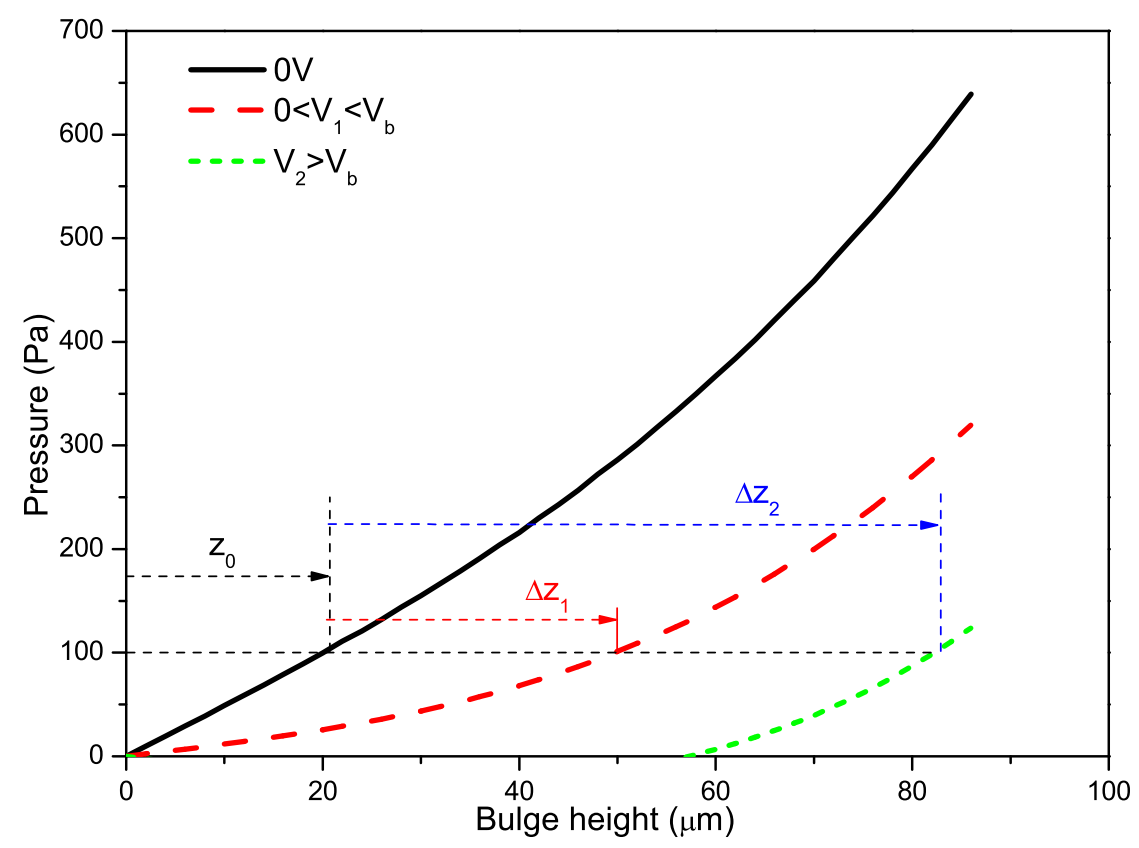

Fig. 3. Calculated pressure-deflection characteristics for three different applied voltages and illustration of the evolution of the equilibrium position for a selected pressure of $100 \mathrm{~Pa}$.

where $t_{0}$ is the initial membrane thickness before deformation. This equation can be substituted into Eq. 5 to take into account the thickness reduction caused by membrane deformation.

Equation 5 does not take into account the hyper-elastic non-linear characteristics of elastomers and assumes a purely Hookean behavior. However, uniaxial compression tests conducted on macroscale PDMS cylinders exhibited a linear behavior for strains up to 10-20\% [8]. In our application, the maximal pressure- and voltage-induced vertical deformation applied to the actuator was up to $15 \%$ of its diameter, which correspond to a surface strain of $8.3 \%$ at most. Hence, these relatively small strains justifiy using a linear stress-strain relation to model our actuators' behavior.

Without any voltage applied, Eq. 5 is the bulge test equation. The bulge test is a well known technique to extract mechanical parameters (Young's Modulus and residual stress) from thin film membranes by applying a pressure on one side of the membrane and recording its deflection [11-13]. We have adapted this technique to soft polymer membranes and have used it to extract the mechanical data of our DEAs [6]. By applying a voltage between the compliant electrodes, the equilibrium position is modified as shown in Fig. 3 for three different applied voltages: 1) $0 \mathrm{~V}$; this is the calculated equilibrium point for a 
membrane representative of our samples with an applied pressure on one side. When a pressure of $100 \mathrm{~Pa}$ is applied to this membrane, it will be deformed by $20 \mu \mathrm{m}$ to reach the equilibrium $z_{0}$. 2) When a voltage is applied the stress state inside the membrane is modified and the deformation for a given pressure is increased by $\left.\Delta z_{1} .3\right)$ If the Voltage is further increased, above the buckling voltage $\left(V_{b}\right)$, the equilibrium position moves to $z_{0}+\Delta z_{2}$. In that case, a deformation is observed even without pressure applied to the membrane. Using the geometrical and mechanical properties of an EAP membrane, Eq. 5 can therefore be used to calculate the expected displacement for every values of distributed load and voltage, or inversely, given a desired output displacement for a loading pressure, the equation can be used to find the mechanical and geometrical parameters needed to meet the expected performance. A similar equation can be derived from the bulge test equation for square membranes of side $a[13]$ :

$$
p=\frac{1}{0.792+0.085 \nu} \frac{E t}{(1-\nu)(a / 2)^{4}} z^{3}+3.393 \frac{\sigma_{x, y} t}{(a / 2)^{2}} z .
$$

From the equation linking the deflection to the applied pressure, one can calculate the displacement from the equilibrium position $\Delta z$ which is caused by the electrostatic force simply by removing the pressure-induced deformation $z_{0}$ (Fig. 3):

$$
\Delta z(p, V)=z(p, V)-z(p, 0)
$$

where $z(p, V)$ is the reciprocal function of Eq. 5 or 7 . Finally, the mechanical work $W$ is calculated by integrating over the surface the displacement $z$ of each surface element $d S$ multiplied by the distributed force $p$ :

$$
W=\int_{z_{0}+\Delta z}^{z_{0}} z(p, V) \cdot p \cdot d S
$$

For circular membranes, and with the hypothesis of deformation in a portion of sphere, it is defined by:

$$
W=\frac{p \cdot \pi \cdot \Delta z\left(\Delta z^{2}+3 \Delta z \cdot z_{0}+3\left(r^{2}+z_{0}^{2}\right)\right)}{6} .
$$

The maximal voltage that can be applied to the actuator is limited by the dielectric breakdown of the elastomer, which varies between 35 and $50 \mathrm{~V} / \mu \mathrm{m}$ for our membranes with ion-implanted electrodes. The maximal distributed force (pressure) is limited by the rupture point of the PDMS, which typically 
occurs for elongations between $400 \%$ and $650 \%$. The membranes can be inflated in the shape of half-spheres without breaking. This corresponds to a pressure of approximately $30 \mathrm{kPa}$. However the simple model presented here cannot be applied for such high pressures/deformations at which the hyperelastic behavior of PDMS cannot be neglected. More complete models have been developed for this situation or for highly prestreched membranes [14].

\section{Fabrication Process and Characterization}

\subsection{Fabrication of Freestanding Membranes}

Samples consist of $30 \mu \mathrm{m}$-thick PDMS layers bonded on silicon ( $\mathrm{Si}$ ) chips with circular and square through-holes of lateral dimension 2 to $3 \mathrm{~mm}$. Both sides of the membrane are implanted at $5 \mathrm{keV}$ with $\mathrm{Au}$ ions with doses in the range of $1-2 \cdot 10^{16}$ atoms $/ \mathrm{cm}^{-2}$.

PDMS (Nusil CF19-2186) is mixed with isooctane to lower its viscosity and spin-coated on a flexible polyvinylidene chloride (PVDC) sheet coated with a photoresist sacrificial layer, which allows easy bonding on a silicon wafer. The PDMS is then cured at at room temperature or at $6{ }^{\circ} \mathrm{C}$ during 48 hours to minimize residual tensile stress, which is very sensitive to curing conditions [6]. Circular and square through-holes of lateral dimensions 2 to $3 \mathrm{~mm}$ are patterned with DRIE on a 4" silicon wafer. The cured PDMS layer is then bonded on the patterned silicon wafer after an oxygen plasma treatment. The assembly is then dipped into acetone to dissolve the sacrificial layer and remove the PVDC transfer substrate. The wafers are diced into chips of $20 \times 20 \mathrm{~mm}^{2}$. Resulting membranes have a Young's Modulus of 0.5-0.6 MPa, a thickness of $22-30 \mu \mathrm{m}$ and a residual tensile stress of $10-40 \mathrm{kPa}$.

\subsection{Implantation}

Implantation for EAP applications needs to meet three criteria : 1) a high ion flux to keep implanting time short, 2) the possibility to work at low energy to limit the penetration of the implanted species in the first few nanometers of the target, and 3) a limited impact on the stiffening of the implanted membranes. Of the different implantation techniques we experimented with, FCVA is the only one meeting the three criteria. Figure 4 shows the principle of FCVA implantation: the plasma gun and substrate lie in a vacuum chamber $\left(p<5 \cdot 10^{-6}\right.$ mbar). A high voltage pulse between the trigger and the cathode initiates the main $600 \mu$ s-arc between the anode and cathode. 


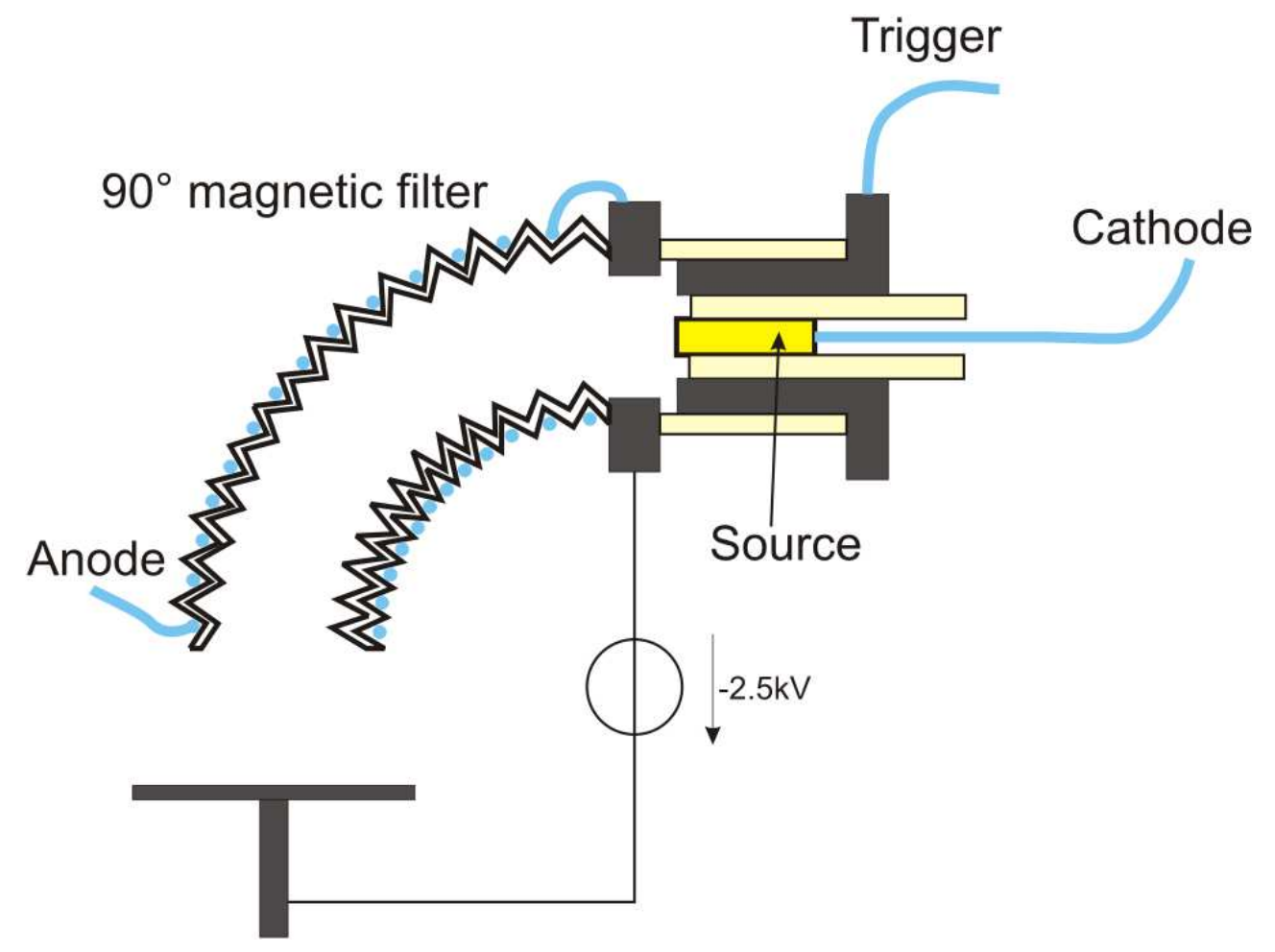

Fig. 4. Schematic representation of FCVA implantation.

Table 1

Charge state of Gold ions generated by vacuum arc [15].

\begin{tabular}{cccc}
\hline Element & $\mathrm{Q}=\mathbf{1}+(\boldsymbol{\%})$ & $\mathbf{2 +}(\boldsymbol{\%})$ & $\mathbf{3 + ( \% )}$ \\
\hline Gold & 14 & 75 & 11 \\
\hline
\end{tabular}

During the pulse, the solid cathode surface is vaporized, which creates metal ions and big, heavy, undesirable macroparticles. They are accelerated by the pressure gradient and enter a $90^{\circ}$ electromagnetic filter consisting of a bellow with a solenoid coil around it that bends the trajectories of the ions. The macroparticles' trajectories are not altered by the electromagnetic field and they collide with the duct walls and are eliminated. At the filter output, the positive ions are accelerated toward the target by polarizing the substrate holder at a negative potential relatively to the output of the filter.

Ion energy and dose are difficult to control precisely in our FCVA. Ion flux is measured with a Faraday cup and depends on wear of the source cathode and its relative positioning to the anode. The energy is not well defined, due to the ions charge distribution (Tab. 1) and dips of the accelerating potential during each pulse caused by the large currents drawn from the source. We use a voltage of $-2.5 \mathrm{kV}$ during implantation and a number of pulses chosen to reach an implanted dose of $1-2 \cdot 10^{16} \mathrm{~cm}^{-2}$, which leads to surface resistivity of $100-1000 \Omega$ /square. 


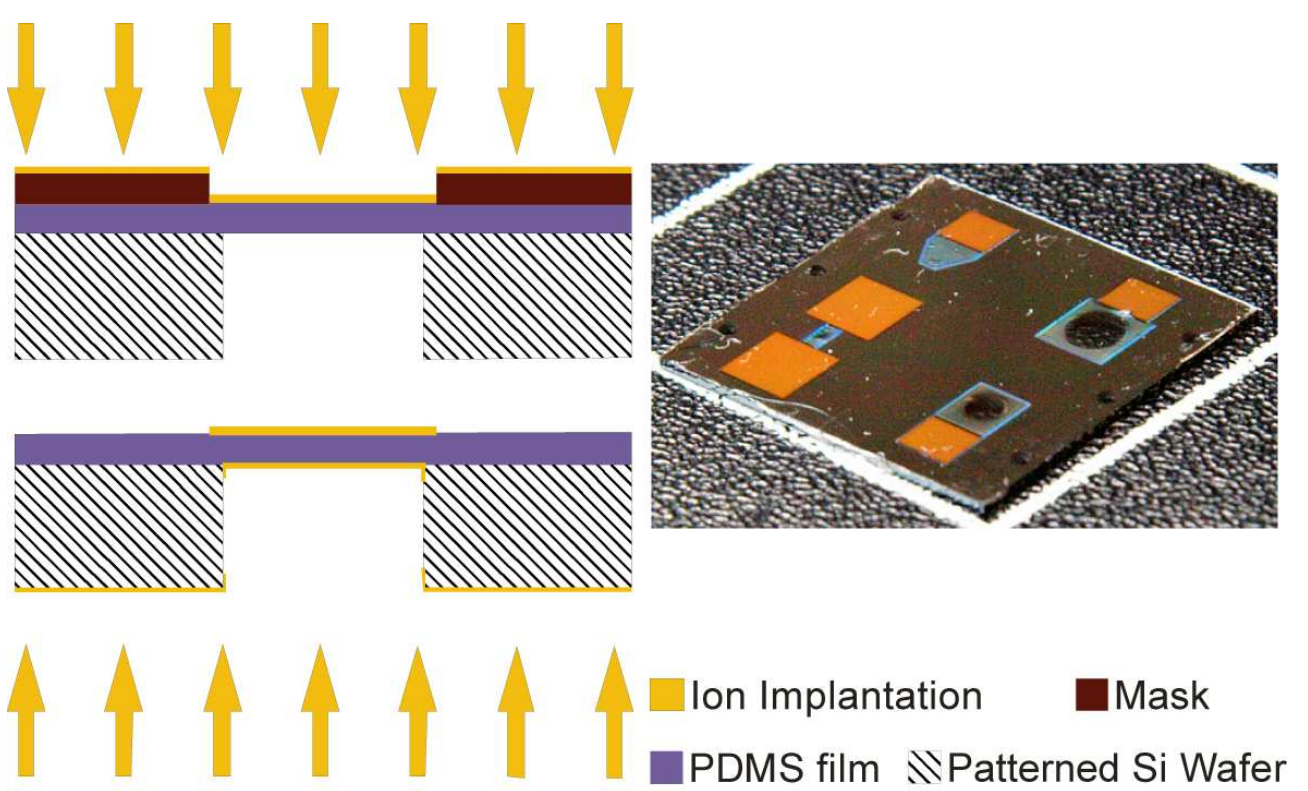

Fig. 5. Left: topside implantation through a shadow mask (top), and backside implantation through the openings in the Si chip (bottom). Right: complete chip with gold pads for electrical contacts.

Topside implantation is conducted through a steel shadow mask to define several independent devices on a single chip. Patterned photoresist can also be used as a mask for implantation if better resolution is needed. Backside implantation is directly conducted through the opening in the silicon chip. An electrical contact is created by the ions between the membrane and the silicon frame, which can act as the backside electrical connection (Fig. 5). A sputtered gold pad is also deposited on the surface of the implanted PDMS close to each membrane to provide the top electrical contact.

\section{Measurements and Results}

\subsection{Mechanical properties}

The mechanical properties are measured on a bulge test setup. The chip with the implanted membranes is mounted on an airtight socket and placed under an optical profiler (Wyko NT1100 from Veeco). A syringe pump is connected to the socket via a large $(300 \mathrm{ml})$ buffer volume. A barometric sensor (Intersema MS5537) with $1 \mathrm{~Pa}$ resolution is connected to the circuit to measure the applied pressure, which is varied from 0 to $1 \mathrm{kPa}$ by steps of $15 \mathrm{~Pa}$. For each pressure step, the central deflection is measured with the profiler, which allows the extraction of the Young's Modulus and residual stress according to Eq. 5 or Eq. 7. These two parameters can then be used to calculate the membrane's 
Table 2

Properties of the membranes before and after implantation. Ion dose is for each electrode.

\begin{tabular}{llllllc}
\hline & $\begin{array}{c}\text { Thickness } \\
(\mu \mathrm{m})\end{array}$ & \multicolumn{2}{c}{ Before Impl. } & Ion Dose & \multicolumn{2}{c}{ After Impl. } \\
& \multicolumn{1}{c}{ E $(\mathrm{MPa})$} & $\sigma(\mathrm{kPa})$ & $\left(\mathrm{cm}^{-2}\right)$ & $\mathrm{E}(\mathrm{MPa})$ & $\sigma(\mathrm{kPa})$ \\
\hline \hline Membrane 1 & 22.5 & 0.52 & 20.9 & $1 \cdot 10^{16}$ & 0.75 & 17.4 \\
\hline Membrane 2 & 29.2 & 1.07 & 48.3 & $1.5 \cdot 10^{16}$ & 2.54 & 42.4 \\
\hline Membrane 3 & 24.4 & 0.98 & 32.2 & $2 \cdot 10^{16}$ & 5.57 & 9.8 \\
\hline
\end{tabular}

deflection for any combination of applied pressure and voltage. The exact same setup and procedure is used to characterize the actuators performance under load and electrical actuation: the pressure-deflection curve is measured for different voltage values, from $0 \mathrm{~V}$ to $800-1000 \mathrm{~V}$.

The mechanical properties of the tested membranes were measured before and after implantation. Two different temperatures were used during polymerization of PDMS and different ion doses were used, which leads to the actuators' properties reported in Tab. 2. Membrane 1 and 3 have a diameter of $3 \mathrm{~mm}$ and membrane 2 has a diameter of $2 \mathrm{~mm}$. Membrane 1 is polymerized at $6{ }^{\circ} \mathrm{C}$, which results in a low Young's Modulus and residual tensile stress. It is also implanted with the lowest dose and the final actuator has mechanical properties relatively close to the unimplanted membrane. Membrane 2 and 3 are polymerized at room temperature and have higher Young's Modulus and residual tensile stress. Mechanical properties of membrane 3, which is implanted with the highest dose, are greatly influenced by the implantation. The Young's Modulus increases by $470 \%$, reflecting the stiffening of the membrane by the $\mathrm{Au}$ ions. The residual stress, however, is decreased by the addition of $\mathrm{Au}$ particle below the polymer's surface, creating a localized compressive stress. This is interesting to lower the buckling voltage (c.f. Eq. 4), as with our process, $V_{b}$ is dominated by $\sigma_{0}$ and not by the E-dependant critical stress.

\subsection{Displacement and mechanical work}

Membrane 1 is the membrane which has the lowest Young's modulus, hence it is easily deformed by Voltage or mechanical loading (Fig. 6). At the dielectric breakdown limit $(800 \mathrm{~V}$ or $35.6 \mathrm{~V} / \mu \mathrm{m})$ the free-strain displacement is $200 \mu \mathrm{m}$ which represents $6.67 \%$ of the actuator's diameter. When the maximal distributed force of $7 \mathrm{mN}$ is applied, the displacement goes from $362 \mu \mathrm{m}$ for $0 \mathrm{~V}$ to $448 \mu \mathrm{m}$ for $800 \mathrm{~V}$, this corresponds to a voltage-induced displacement of $86 \mu \mathrm{m}$ ( $2.87 \%$ of the actuator's diameter ), or a volume change of 326 nl. The analytical model presented in $\S 2$ shows an excellent agreement with 


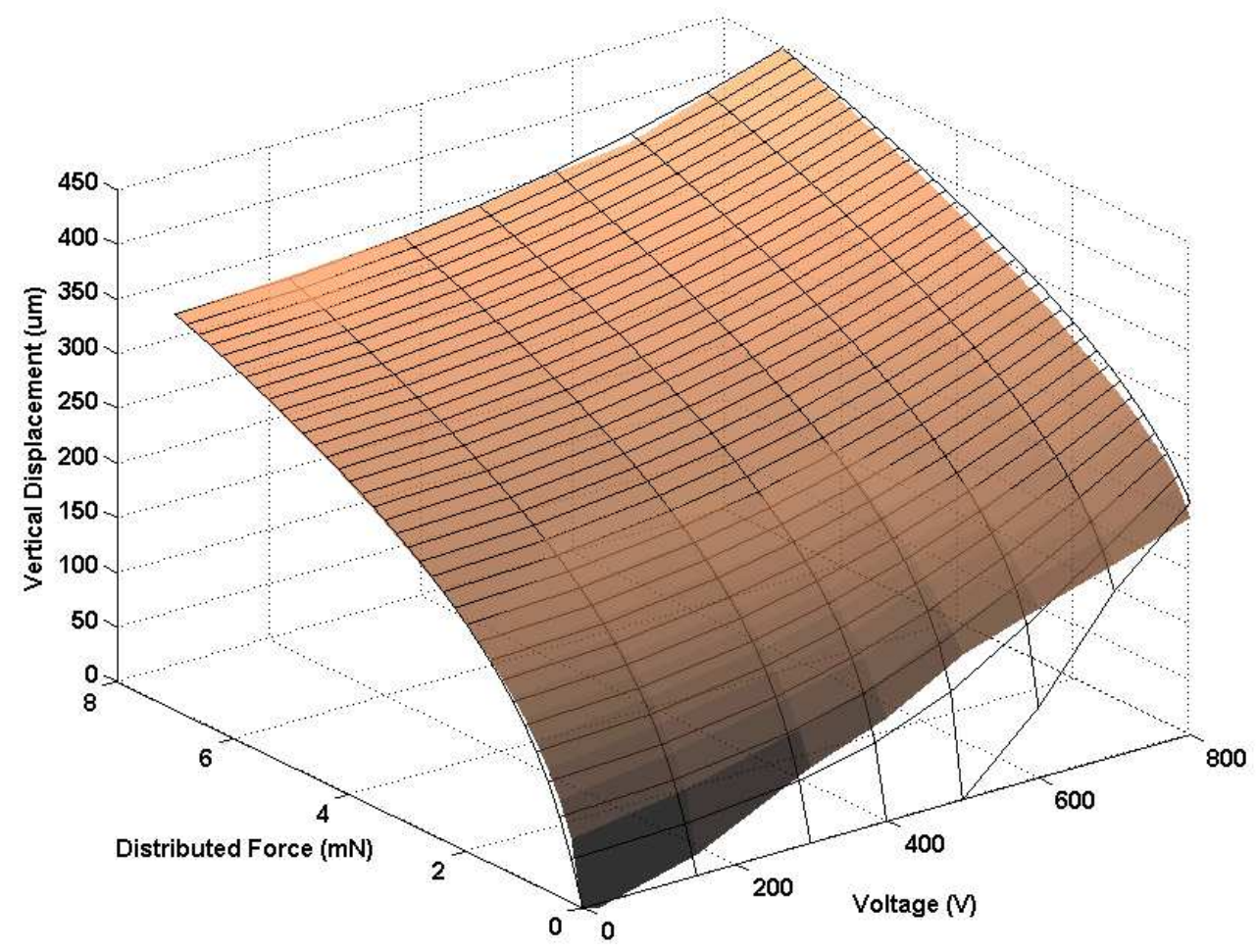

Fig. 6. Vertical displacement of the center of membrane 1 for voltages between 0 and $800 \mathrm{~V}$ (dielectric breakdown), and applied distributed force between 0 and 7 $\mathrm{mN}$ (0-990 Pa). Wireframe: Theoretical model. Plane: datapoints.

the data points. The biggest discrepency is observed for the unloaded actuator: buckling as predicted by the model is not observed in the measurement because of initial deformation of the membrane which is not perfectly flat. Consequently, vertical displacement is observed even if the critical buckling stress is not reached.

Membrane 2 has a smaller diameter, is thicker and has a higher residual tensile stress. All three factors contribute to raise the buckling voltage, which has not yet been reached for a voltage of $1000 \mathrm{~V}$ (Fig. 7), and to reduce the vertical displacement. For a voltage of $1000 \mathrm{~V}(34.2 \mathrm{~V} / \mu \mathrm{m})$ and no applied mechanical load, the vertical displacement is $65 \mu \mathrm{m}$, which is only $3.25 \%$ of the membrane's diameter, and the voltage-induced displacement (Eq. 8) with the maximal applied distributed force $(2.75 \mathrm{mN}$ or $875 \mathrm{~Pa})$ at $1000 \mathrm{~V}$ is $31 \mu \mathrm{m}$ or $1.55 \%$.

Membrane 3 has been implanted with the highest dose of gold and has the highest Young's Modulus, but it also has the lowest residual stress, resulting in a low buckling voltage. However, the higher membrane's stiffness reduces the amplitude of the voltage-induced displacement $\Delta z$ (Fig. 8). This demonstrates that reducing the residual stress with a higher implantation dose is not an interesting solution, because it causes a too important stiffening of the membrane. The best performance is obtained with membranes that have a 


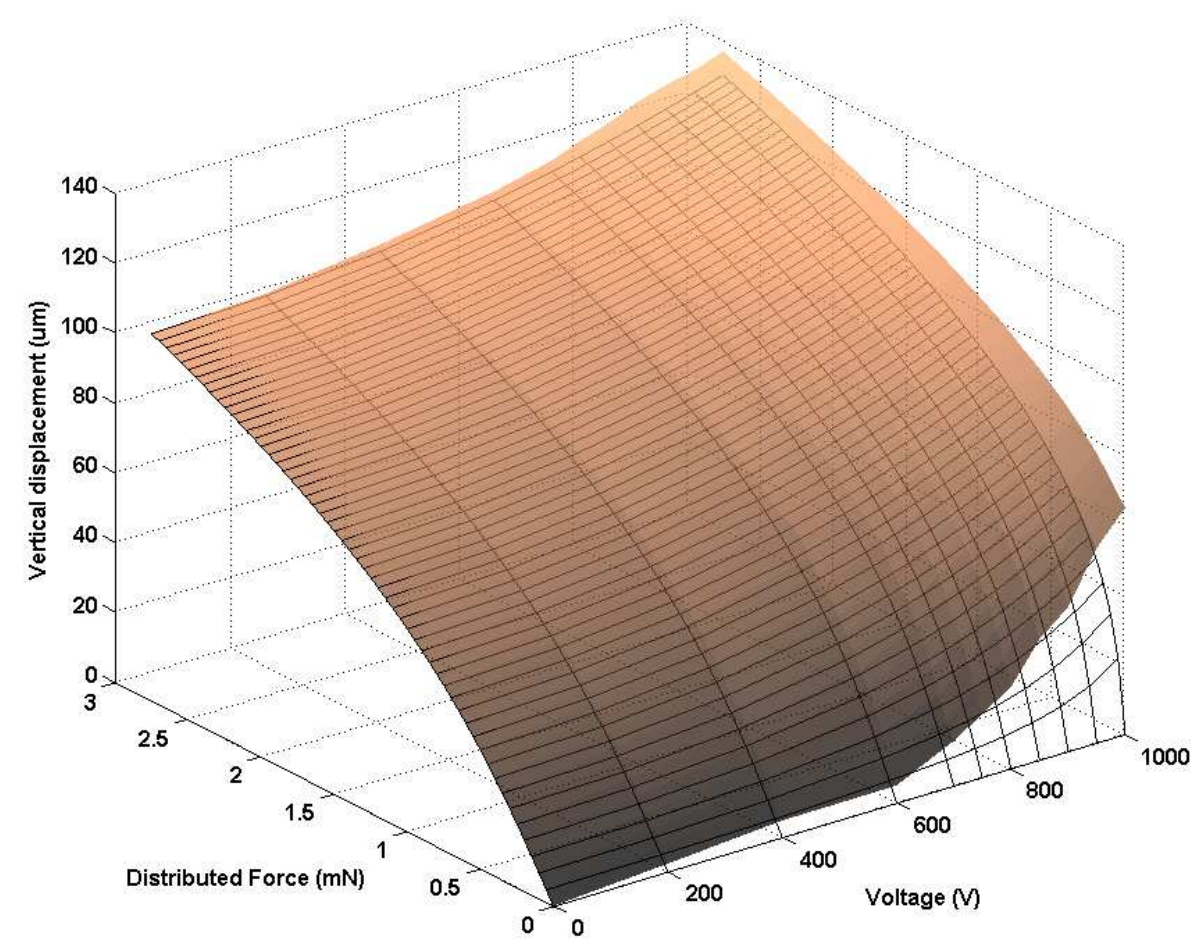

Fig. 7. Vertical displacement of the center of membrane 2 for voltages between 0 and $1000 \mathrm{~V}$, and applied distributed force between 0 and $2.75 \mathrm{mN}(0-875 \mathrm{~Pa})$. Wireframe: Theoretical model. Plane: datapoints.

very low internal stress before the creation of electrodes, and with the lowest possible dose which is sufficient to create a conductive surface, thus having a small impact on the membrane's Young's Modulus. This correspond to doses in the region of the percolation limit, around which a small dose change results in an important modification of electrical resistivity and membrane's stiffening. Between $1 \cdot 10^{16}$ and $2 \cdot 10^{16} \mathrm{~cm}^{-2}$, the relative stiffening increases from $44 \%$ to $470 \%$ and the surface resistivity decreases from $1 k$ to $100 \Omega /$ square: a change of one order of magnitude for a dose change of a factor 2 .

Due to its larger vertical displacement, membrane 1 is able to produce a higher mechanical work with a maximum of 320 nJ per cycle (Fig. 9). Membrane 2, with its smaller size, produces the smallest amount of work, but also occupies a smaller surface on the chip. The work density (work per volume unit) is a better representation to compare the three different membranes (Fig 10). As expected, the membrane implanted with the lowest dose exhibits the best performance. Membrane 2, with its intermediate gold dose performs better than the highly implanted membrane for applied pressure larger than $400 \mathrm{~Pa}$. For lower pressures, the mechanical work output is limited by the relatively high buckling voltage due to the smaller size and the highest residual tensile stress. 


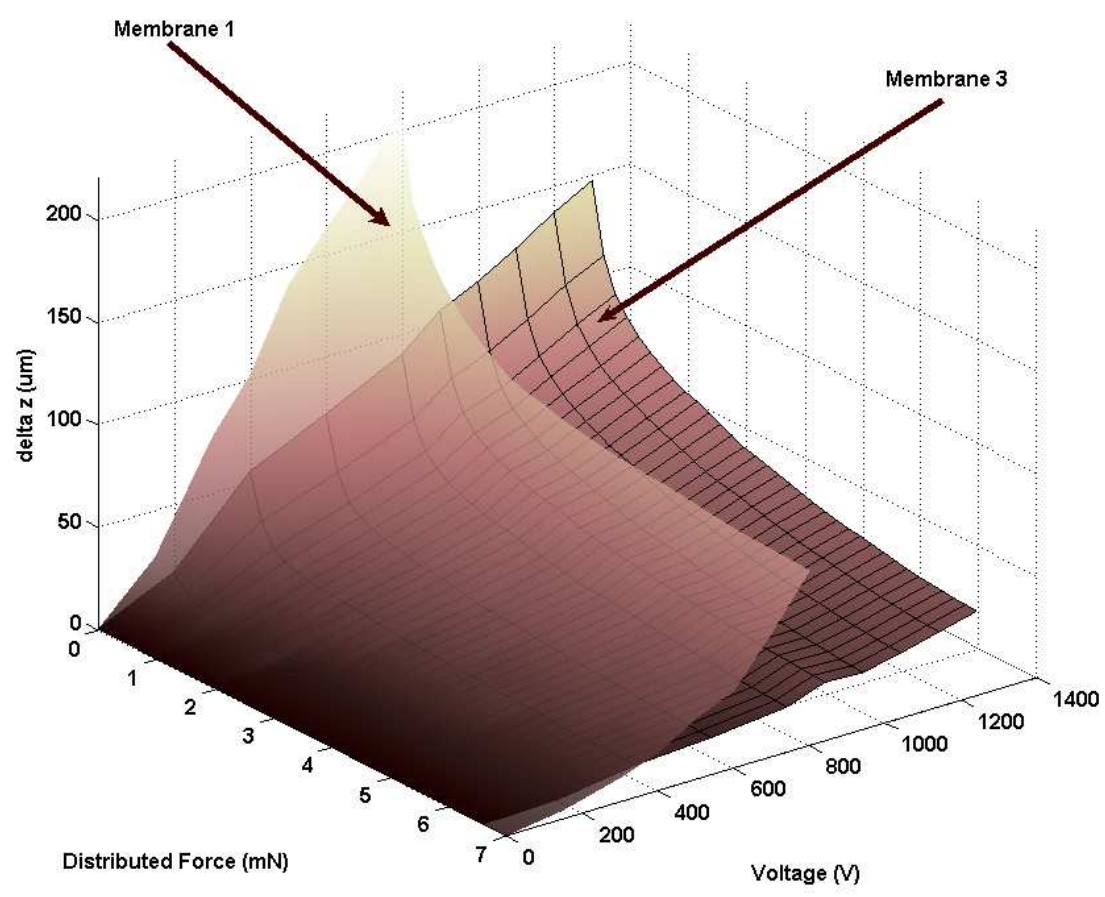

Fig. 8. Voltage-induced displacement $\delta z=z(p, V)-z(p, 0)$ measured for membrane 1 and 3 . Due to a higher Young's modulus, membrane 3 exhibits a smaller vertical displacement.

\subsection{Transient and Frequency Response}

Transient response has been measured with a doppler laser vibrometer (Polytec MSV-400). A square signal between 0 and 490V was applied to the actuator at $1 \mathrm{~Hz}$ and the deflection of the membrane's center was recorded as a function of time (Fig. 11).

The response time of the actuator was measured during the fall time only due to the impossibility to get a fast enough rising edge transition with our high voltage source. The membrane used for the measurements has mechanical properties very similar to those of membrane 1 (Tab. 2). Figure 11 shows the transient response of the membrane to a time-variant signal. The rising edge shows buckling with a rapid jump to a height of $10 \mu \mathrm{m}$. Then the membrane continues to move upward more slowly. The fall response time defined as the time taken by the actuators to move from $90 \%$ to $10 \%$ of the full step height. For our tested membrane, the response time is $145 \mathrm{~ms}$, which is higher than expected given the mechanical resonance frequency of $2 \mathrm{kHz}$. It may be due to a high contact resistance between the implanted electrode and the contact wire or by the viscoelastic response of the polymer, which has yet to be fully characterized. Solvent added to the PDMS for the spin-coating propably has 


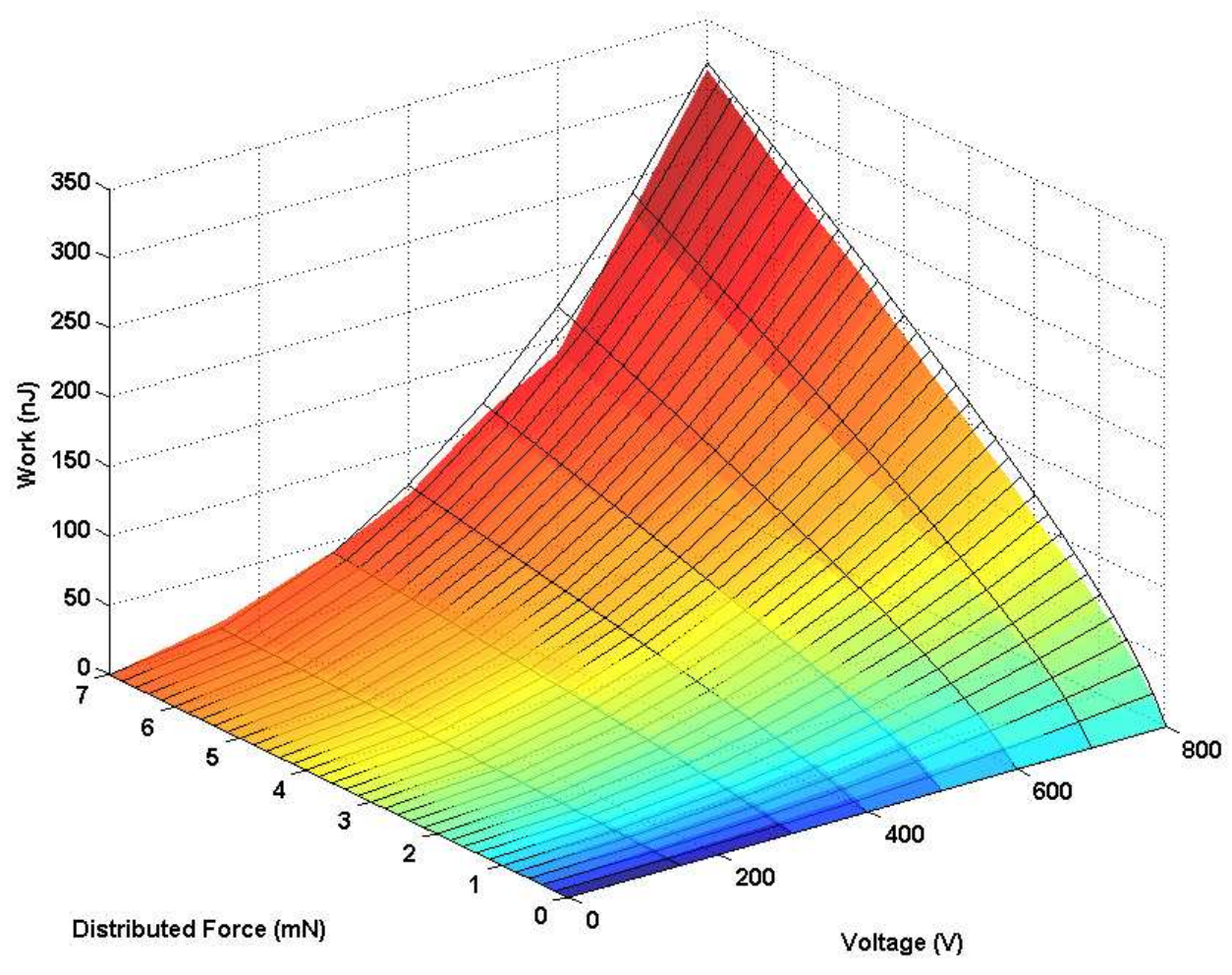

Fig. 9. Mechanical work of membrane 1 as a function of applied voltage and distributed force. Wireframe: Theoretical model. Plane: datapoints.

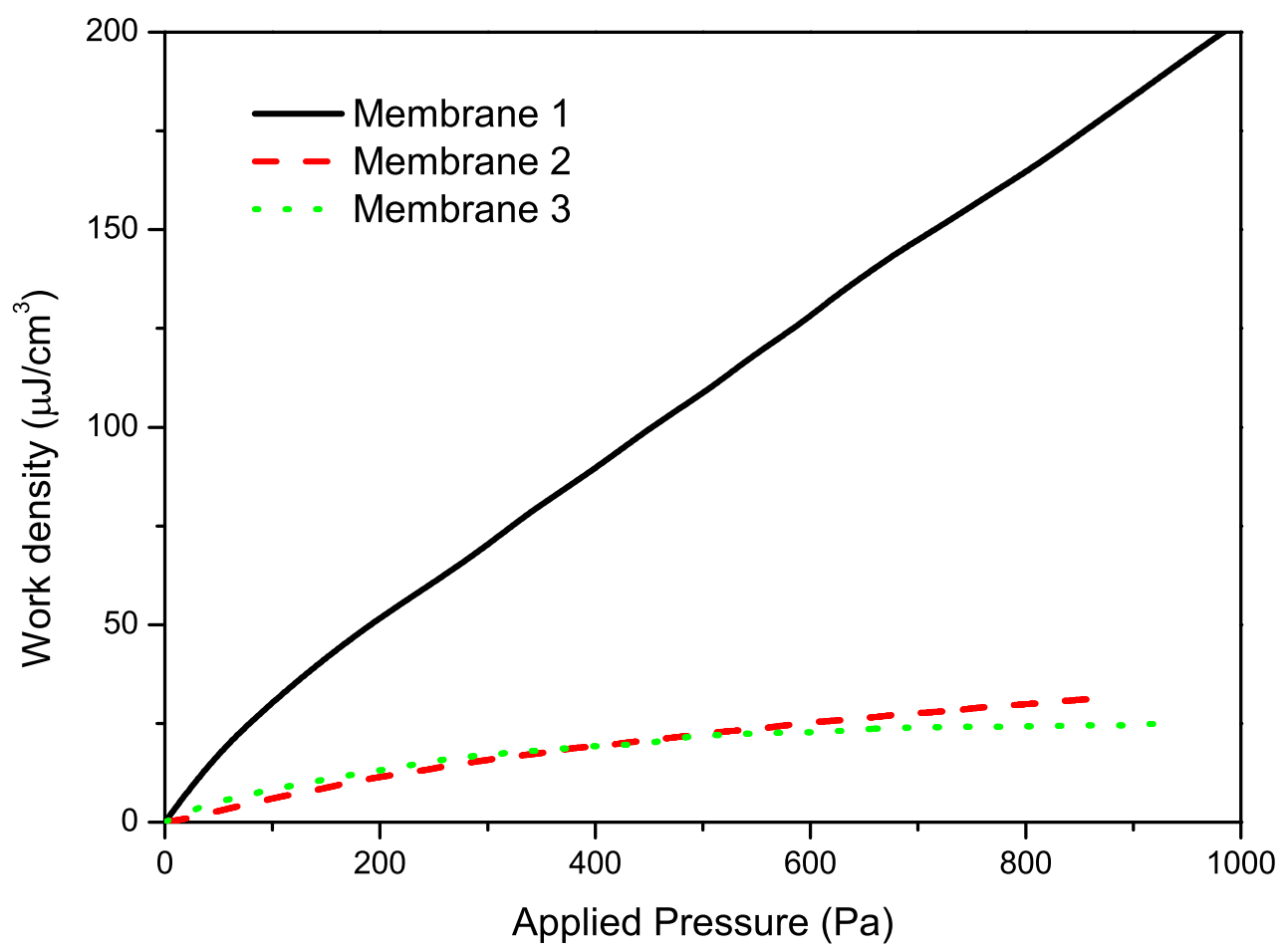

Fig. 10. Mechanical work density for the three membranes for an applied voltage of $800 \mathrm{~V}$. The electrostatic energy stored in the dielectric is not taken into account 


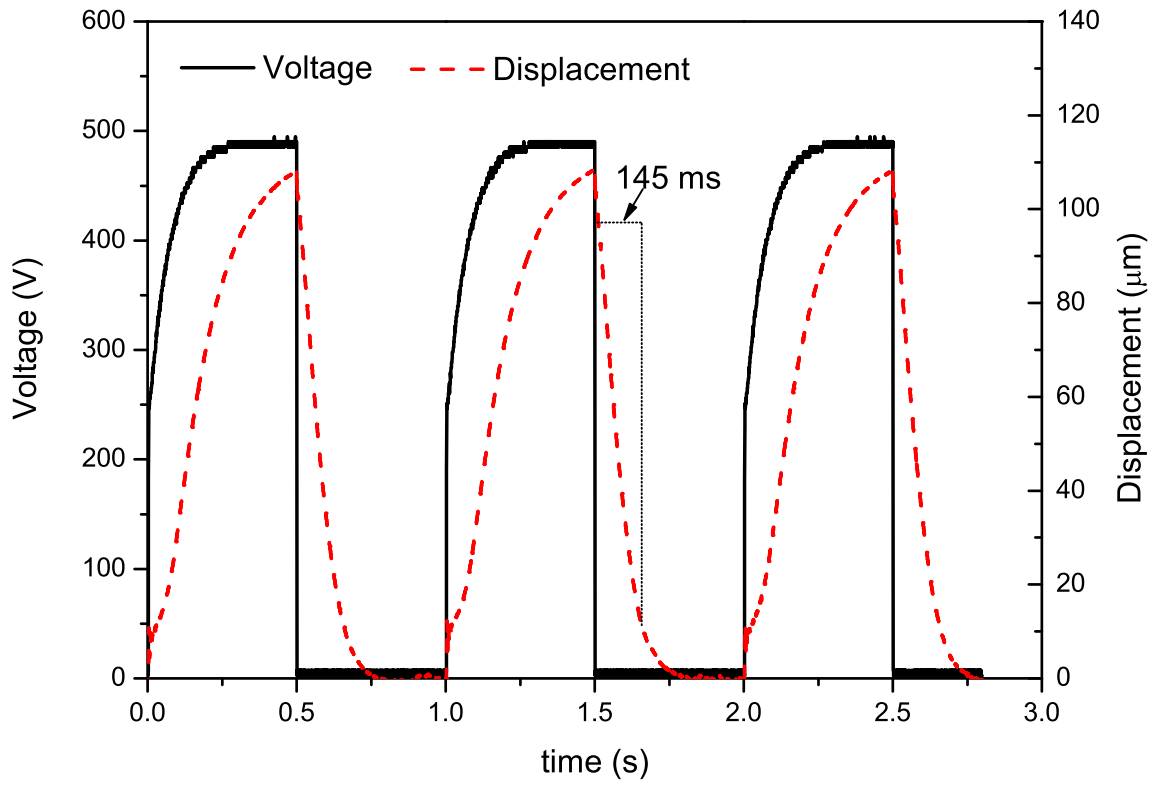

Fig. 11. Actuator's response (dashed line) to voltage steps of 490V (solid line). Measurement of the displacement of the membrane's center.

an influence on the viscoelastic response of PDMS.

Aging of the response was measured by applying a $2 \mathrm{~Hz}$ square signal between 0 and $200 \mathrm{~V}$, and measuring maximum displacement per cycle as a function of cycle number. The initial displacement was $34 \mu \mathrm{m}$ and decreased to $23 \mu \mathrm{m}$ after 80 thousands cycles.

\section{Discussion}

The measurements presented here show that the membrane's mechanical properties, both before and after implantation, determine the performance of the micro-actuator. A low buckling voltage allows working with lower voltages and can be achieved by having a large diameter, a thin dielectric layer and low residual stress. The compliance of the electrodes is also crucial as can be seen by comparing the response of membrane 1 and 3 , which have similar properties before implantation. However, membrane 3, with its higher implanted gold dose, is only able to produce $1 / 8^{\text {th }}$ of membrane 1 's mechanical work.

Although simplified and straightforward, the analytical model presented in $\S 2$ shows an excellent agreement with the measurements and can easily be 


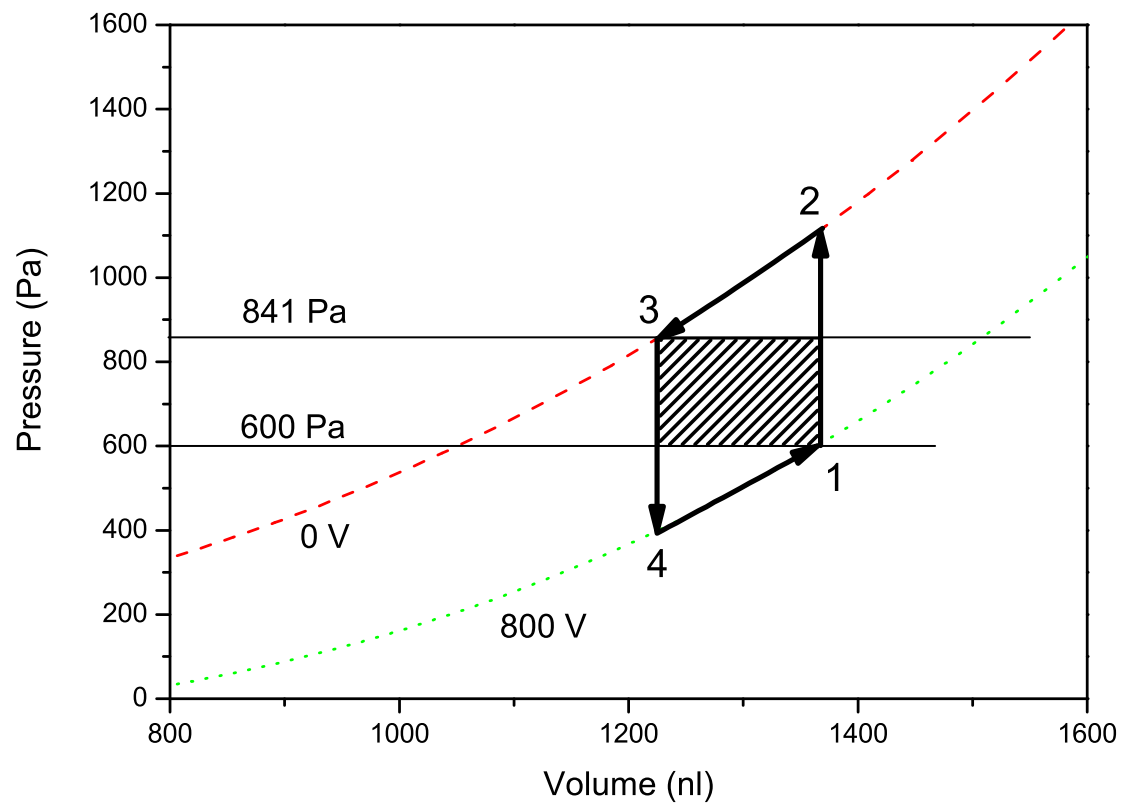

Fig. 12. Example of a theoretical work cycle of a DEA used as a micropump and actuated between 0 and $800 \mathrm{~V}$. The input pressure point is chosen at $600 \mathrm{~Pa}$, and the output pressure is chosen to maximize the potential energy gain (hatched rectangle).

used for the dimensioning of non-prestreched EAP diaphragms subjected to a distributed load, provided the mechanical and geometrical parameters of the actuators are known. The geometry of the actuator is process-related and can be controlled or measured. The mechanical properties are also process-related and may be harder to control. However, they can be measured with a bulge test in the case of freestanding membranes.

Taking a DEA with the parameters of membrane 1, one can use the analytical model to calculate the performance one could expect from such an actuator if used as a micropump with hypothetical perfect inlet and outlet valves (Fig. 12), in a development similar to what Tews et al. did for larger actuators. [16]. Assuming an inlet pressure $\left(P_{i n}\right)$ of $600 \mathrm{~Pa}$, and an actuation voltage of 800 $\mathrm{V}$, the membrane's equilibrium position is represented by the point 1 . If the voltage is removed, the membrane compresses the fluid and the pressure rises to point 2 . The liquid can leave the chamber until the pressure decreases to the outlet pressure $\left(P_{\text {out }}\right)$, at which time point 3 is reached. The membrane is then reactivated, which lowers the pressure in the chamber (point 4) and allows the liquid to enter the chamber to go back to the initial position. Total work done per cycle is represented by the surface enclosed by the loop and can be divided in one rectangle (hatched surface) and two triangle-like shapes. The rectangle represents the potential energy transmitted to the fluid by the pressure gain, 
and the triangles reflects the kinetic energy added to incoming and outgoing fluid, which is lost in most pumps designs (at least for the energy added to the fluid entering the pump). Total work is maximized if the two pressures are equal, but in that case, there is only kinetic energy created, half of which at least is lost and dissipated. Given a working point for $P_{\text {in }}(378 \mu \mathrm{m}, 600 \mathrm{~Pa})$ on the activated curve, there is an optimal output point $\left(z_{\text {out }}, P_{\text {out }}\right)$ which will maximize the potential energy transmitted to the fluid. This can easily be calculated by maximizing

$$
E_{\text {pot }}=\left(\operatorname{Vol}\left(z_{\text {in }}\right)-\operatorname{Vol}\left(z_{\text {out }}\right)\right)\left(\mathrm{p}\left(z_{\text {out }}, 0 \mathrm{~V}\right)-\mathrm{p}\left(z_{\text {in }}, 800 \mathrm{~V}\right)\right)
$$

where $\operatorname{Vol}(z)$ is the volume of the chamber when the membrane is deformed to a height $z$, and $\mathrm{p}(z, V)$ is the equilibrium pressure for a displacement $z$ and an applied voltage $V$ (Eq. 5). By moving the input point to a lower pressure and selecting the output point to maximize the potential energy, the ratio $E_{\text {pot }} / E_{\text {kin }}$ increases, but the value of the potential energy per cycle is decreased. The opposite is observed if the pumping loop is moved toward higher pressure.

For this example, the optimal output height is $338.4 \mu \mathrm{m}$, which correspond to an output pressure of $841 \mathrm{~Pa}$, and a pumped volume of $148.3 \mathrm{nl}$ per cycle. The mechanical work converted to potential energy is $35.7 \mathrm{~nJ}$ per cycle, the kinetic energy of the fluid leaving the pump is $19.7 \mathrm{~nJ}$, and the kinetic energy dissipated by the fluid entering the pump is $15.8 \mathrm{~nJ}$. The total mechanical work produced by the pump is $71.2 \mathrm{~nJ}$ per cycle. For an equivalent electrostatic force, the work divided by the volume of the membrane is approximately 15 times smaller than what Tews et al. obtained for the same polymer. Direct comparison is difficult, because our membranes have a thickness over surface ratio 40 times larger than those of Tews et al. However, it should be pointed out that the pressure difference has been conserved during miniaturization, and that it is the pumped volume per cycle which is greatly reduced.

\section{Conclusion}

Metal ion implantation on the surface of soft polymers has been shown to create compliant electrodes for DEAs. This technique opens new perspectives for the miniaturization of DEAs, which was held back due to the lack of an applicable solution to manufacture clean and patternable compliant electrodes of dimensions less than $1 \mathrm{~cm}^{2}$. DEAs fabricated with our process achieved unloaded vertical displacement up to $7 \%$ of their lateral dimension and the measured data are in very good agreement with our analytical model for the range of voltages and pressures used. Furthermore, the model can also be 
applied to larger non-prestrained DEAs, as long as the vertical displacement does not exeed half of the membrane's diameter. Larger displacement could be achieved with our actuators by reducing the thickness over surface ratio of the membranes. The results presented in this paper demonstrate the high influence of the stiffening of the membrane due to the electrode on the performance of the actuators, and the importance of having electrodes as compliant as possible. Ion implantation is also an interesting alternative to conventional carbon-based electrodes for macroscale EAPs, for it is cleaner to work with and it does not add mass to the PDMS membrane. At doses close to the percolation threshold, ion implantation does not significantly alter the transparency of the PDMS, which opens up a broad field of applications for which optical transmission through the actuator is desirable.

\section{Acknowledgments}

The authors wish to thank the COMLAB staff for help with device fabrication, and acknowledge financial support from the Swiss National Science Foundation grant \#20021-111841 and from the EPFL.

\section{Biographies}

Samuel Rosset studied microengineering at the Ecole Polytechnique Fédérale de Lausanne and received his MSc degree in 2004. In 2005, he joined Prof. H.R. Shea's group as a PhD student and is working on miniaturized electroactive polymer actuators. His current activities involve the characterization of loaded DEAP micro-actuators and setting up an experimental FCVA implantation system.

Muhamed Niklaus received his master in physics at EPFL 2005. During his master project he developed a theoretical model to describe configurations and scaling properties of DNA. Actually he is concentrated in the field of ion implantation and is developing the methodology to analyze elastomer implanted with metallic ions. He masters many analysis equipments such as SPM, SEM, TEM etc.

Philippe Dubois graduated in electrical engineering from the Neuchâtel University of applied science in 1991, and he received in 1998 a diploma of electronics/physics from the University of Neuchâtel. In 2003 he obtained his Ph.D. on micromachined active valves and tribological studies in the group of professor de Rooij at the IMT, University of Neuchâtel. He is finishing a post-doctoral work focused on liquid valves and directional acceleration sensors, and leads 
projects in the group of professor de Rooij as part time researcher. Presently he leads researches on polymer actuators in the group of professor Shea in the field of microsystems for space at the EPFL.

Dr. Shea has a Ph.D. (1997) and a M.A. (1993) in physics from Harvard University, and a B.Sc. (1991) in physics from McGill University. After 2 years as a post-doctoral fellow at IBM's T.J. Watson Research Center he joined Lucent Technologies' Bell Labs in Murray Hill, NJ, USA, first as a member of technical staff (1999-2001), then (2001-2004) as the technical manager of the Microsystems Technology group. Since April 2004, he is an assistant professor at the EPFL in Lausanne, Switzerland, with a focus on ultra-reliable MEMS for space applications. Research interests include nanosatellites, polymer MEMS, ion propulsion, and the reliability and accelerated testing of silicon and polymer based microsystems.

\section{References}

[1] S. Ashley, Artificial muscles, Sci. Am. 289 (4) (2003) 52-59.

[2] Y. Bar-Cohen, Electro-active polymers: Current capabilities and challenges, in: Proc. of SPIE, Vol. 4695, 2002, pp. 1-7.

[3] R. E. Pelrine, R. D. Kornbluh, J. P. Joseph, Electrostriction of polymer dielectrics with compliant electrodes as a means of actuation, Sens. Actuators A: Phys. 64 (1) (1998) 77-85.

[4] F. Carpi, P. Chiarelli, A. Mazzoldi, D. De Rossi, Electromechanical characterisation of dielectric elastomer planar actuators: comparative evaluation of different electrode materials and different counterloads, Sens. Actuators A: Phys. 107 (1) (2003) 85-95.

[5] B. O'Brien, J. Thode, I. Anderson, E. Calius, E. Haemmerle, S. Xie, Integrated extension sensor based on resistance and voltage measurement for a dielectric elastomer, in: Proc. of SPIE, Vol. 6524, 2007, pp. 15-1 - 15-11.

[6] S. Rosset, M. Niklaus, P. Dubois, M. Dadras, H. Shea, Mechanical properties of electroactive polymer microactuators with ion-implanted electrodes, in: Proc. of SPIE, Vol. 6524, 2007, pp. 10-1 - 10-11.

[7] A. Pimpin, Y. Suzuki, N. Kasagi, Micro electrostrictive actuator with metal compliant electrodes for flow control applications, in: IEEE Intl. conf. Microelectromech. Syst., 2004, pp. 478-481.

[8] P. Dubois, S. Rosset, S. Koster, J. Stauffer, S. Mikhailov, M. Dadras, N.-F. de Rooij, H. Shea, Microactuators based on ion implanted dielectric electroactive polymer (eap) membranes, Sens. Actuators A: Phys. 130-131 (2006) 147-154. 
[9] P. Dubois, S. Rosset, M. Niklaus, M. Dadras, H. Shea, Voltage control of the resonance frequency of dielectric electroactive polymer (deap) membranes, submitted to IEEE J. Microelectromech. Syst., 2007

[10] W. C. Young, Roark's Formulas for Stress and Strain, 6th Edition, McGrawHill, New York, 1989.

[11] B. E. Alaca, J. C. Selby, M. T. A. Saif, H. Sehitoglu, Biaxial testing of nanoscale films on compliant substrates: Fatigue and fracture, Rev. Sci. Instrum. 73 (8) (2002) 2963-2970.

[12] M. Small, W. D. Nix, Analysis of the accuracy of the bulge test in determining the mechanical properties of thin films, J. Mater. Res. 7 (6) (1992) 1553-1563.

[13] V. Paviot, J. Vlassak, W. Nix, Measuring the mechanical properties of thin metal films by means of bulge testing of micromachined windows, in: Mater. Res. Soc. Symp. Proc., Vol. 356, 1995, pp. 579-584.

[14] N. Goulbourne, E. Mockensturm, M. Frecker, A nonlinear model for dielectric elastomer membranes, J. Appl. Mech. 72 (6) (2005) 899-906.

[15] I. G. Brown, X. Godechot, Vacuum arc ion charge-state distributions, IEEE Trans. Plasma Sci. 19 (5) (1991) 713-717.

[16] A. M. Tews, K. L. Pope, A. J. Snyder, Pressure-volume characteristics of dielectric elastomers diaphragms, in: Proc. of SPIE, Vol. 5051, 2003, pp. 159169. 


\section{List of Figures}

1 Dielectric EAP (DEAP) principle. When a voltage is applied to the electrodes (typically up to $1 \mathrm{kV}$ ), the electrostatic pressure squeezes the elastomer dielectric (right side). The volume of the dielectric being quasi constant, the whole structure stretches in the case of free boundary conditions (from $[6])$.

2 Calculated critical buckling stress for circular membranes of different radius and thickness for an elastomer with $\mathrm{E}=0.5$ $\mathrm{MPa}$, and a Poisson coefficient of 0.5

3 Calculated pressure-deflection characteristics for three different applied voltages and illustration of the evolution of the equilibrium position for a selected pressure of $100 \mathrm{~Pa}$.

4 Schematic representation of FCVA implantation.

5 Left: topside implantation through a shadow mask (top), and backside implantation through the openings in the Si chip (bottom). Right: complete chip with gold pads for electrical contacts.

$6 \quad$ Vertical displacement of the center of membrane 1 for voltages between 0 and $800 \mathrm{~V}$ (dielectric breakdown), and applied distributed force between 0 and $7 \mathrm{mN}$ (0-990 Pa). Wireframe: Theoretical model. Plane: datapoints.

$7 \quad$ Vertical displacement of the center of membrane 2 for voltages between 0 and $1000 \mathrm{~V}$, and applied distributed force between 0 and $2.75 \mathrm{mN}(0-875 \mathrm{~Pa})$. Wireframe: Theoretical model. Plane: datapoints.

$8 \quad$ Voltage-induced displacement $\delta z=z(p, V)-z(p, 0)$ measured for membrane 1 and 3. Due to a higher Young's modulus, membrane 3 exhibits a smaller vertical displacement.

9 Mechanical work of membrane 1 as a function of applied voltage and distributed force. Wireframe: Theoretical model. Plane: datapoints.

10 Mechanical work density for the three membranes for an applied voltage of $800 \mathrm{~V}$. The electrostatic energy stored in the dielectric is not taken into account 
11 Actuator's response (dashed line) to voltage steps of $490 \mathrm{~V}$ (solid line). Measurement of the displacement of the membrane's center.

12 Example of a theoretical work cycle of a DEA used as a micropump and actuated between 0 and $800 \mathrm{~V}$. The input pressure point is chosen at $600 \mathrm{~Pa}$, and the output pressure is chosen to maximize the potential energy gain (hatched rectangle). 


\section{List of Tables}

1 Charge state of Gold ions generated by vacuum arc [15]. $\quad 9$

2 Properties of the membranes before and after implantation. Ion dose is for each electrode. 